\title{
Effects of intracerebroventricular administration of CGRP on fear memory in mice
}

\author{
Shuta Mishima, Ami Otsuka, Naoya Hashikawa, Narumi Hashikawa-Hobara
}

Okayama University of Science, Japan

Calcitonin-gene related peptide (CGRP) is a 37-amino acid neuropeptide, which plays a critical role in the central nervous system. In recently, we have reported that CGRP evokes extinction of hippocampus-dependent fear memory when intracerebroventricular (i. c. v.) administration to mice. In the present study, we evaluated whether CGRP modulates level of dopamine in mice hippocampus. Dopamine has been shown to be critical for many processes that drive learning memory, motivation and memory consolidation. CGRP injections ( $0.5 \mathrm{nmol})$ shortened the avoidance latency in passive avoidance test, and also reduced freezing time in contextual fear learning test. In contrast, CGRP i. c. v. decreased the level of dopamine in hippocampus. Furthermore, the release of dopamine level, which was induced by high potassium depolarization, was also decreased in hippocampal cell line, HT22 cells. Meanwhile, we performed microarray analysis of hippocampal gene expression measured $3 \mathrm{hr}$ after CGRP i. c. v. administration in mice. Microarray analysis was given the attenuate Slc6a3 (dopamine transporter), Nt3 (Neurotrophin 3) by CGRP administration. Contrary to this, CGRP enhanced the level of Bdnf (brain-derived neurotrophic factor), Fos, Gad2 (Glutamate decarboxylase; GAD65) and Npas4. These results suggested CGRP contribute to change dopamine level and modulate some genes expression through regulate fear memory formation. 